\title{
Temperature-driven nucleation of ferromagnetic domains in FeRh thin films
}

\author{
C. Baldasseroni, ${ }^{1, a)}$ C. Bordel, ${ }^{2,3}$ A. X. Gray, ${ }^{4,5,6}$ A. M. Kaiser, ${ }^{4,5,7}$ F. Kronast, ${ }^{8}$ \\ J. Herrero-Albillos, ${ }^{8,9,10}$ C. M. Schneider, ${ }^{7}$ C. S. Fadley, ${ }^{4,5}$ and F. Hellman ${ }^{1,5}$ \\ ${ }^{1}$ Department of Materials Science and Engineering, University of California Berkeley, Berkeley, California \\ 94720, USA \\ ${ }^{2}$ Department of Physics, University of California, Berkeley, Berkeley, California 94720, USA \\ ${ }^{3}$ GPM, UMR CNRS 6634, Université de Rouen, Av. de l'Université - BP12, 76801 St Etienne du Rouvray, \\ France \\ ${ }^{4}$ Department of Physics, University of California, Davis, California 95616, USA \\ ${ }^{5}$ Materials Sciences Division, Lawrence Berkeley National Laboratory, Berkeley, California 94720, USA \\ ${ }^{6}$ Stanford Institute for Materials and Energy Science, SLAC National Accelerator Laboratory, Menlo Park, \\ California 94029, USA \\ ${ }^{7}$ Peter-Grünberg-Institut PGI-6, Forschungszentrum Jülich GmbH, 52425 Jülich, Germany \\ ${ }^{8}$ Helmholtz-Zentrum Berlin für Materialien und Energie GmbH, Albert-Einstein-Straße 15, D-12489 Berlin, \\ Germany \\ ${ }^{9}$ Centro Universitario de la Defensa, Carretera de Huesca s/n, E-50090 Zaragoza, Spain \\ ${ }^{10}$ Instituto de Ciencia de Materiales de Aragón and Departamento de Física de la Materia Condensada, \\ CSIC - Universidad de Zaragoza, Pedro Cerbuna 12, E-50009 Zaragoza, Spain
}

(Received 25 April 2012; accepted 8 June 2012; published online 25 June 2012)

\begin{abstract}
The evolution of ferromagnetic (FM) domains across the temperature-driven antiferromagnetic (AF) to FM phase transition in uncapped and capped epitaxial FeRh thin films was studied by $\mathrm{x}$-ray magnetic circular dichroism and photoemission electron microscopy. The coexistence of the AF and FM phases was evidenced across the broad transition and the different stages of nucleation, growth, and coalescence were directly imaged. The FM phase nucleates into single domain islands and the width of the transition of an individual nucleus is sharper than that of the transition in a macroscopic average. (C) 2012 American Institute of Physics. [http://dx.doi.org/10.1063/1.4730957]
\end{abstract}

Equiatomic FeRh is a unique material that undergoes a first-order antiferromagnetic (AF) to ferromagnetic (FM) transition just above room temperature (near $350 \mathrm{~K}) .{ }^{1-4}$ This phase transition can be driven by temperature or magnetic field and is coupled to a lattice expansion. Current investigations of this unique transition range from the fundamental understanding of the origin and nature of the transition ${ }^{5}$ to applications associated with the transition such as a giant magnetocaloric effect and a giant volume magnetostriction. ${ }^{6,7}$ FeRh has been studied in the bulk for over 50 years and most recently in thin film form, ${ }^{8-10}$ where the transition temperature has been shown to be sensitive to changes in composition and substrate-induced strain as well as structural and chemical order. FeRh thin films are also a promising candidate for heat-assisted magnetic recording in an exchange-spring system with a hard magnetic layer (for example, FePt). ${ }^{11-13}$ While the first-order character of the transition has been established, the nucleation and growth of both FM (upon heating) and AF (upon cooling) domains and the coexistence of the two phases expected of a first-order transition have never been directly observed. Two previous studies were done on FeRh, both by magnetic force microscopy ${ }^{14,15}$ but they were limited by lack of temperature control which prevented a study of the nucleation and growth across the full transition. Moreover, these domain nucleation and growth processes are crucial to the proposed use and are affected by the surfaces and interfaces of the film.

\footnotetext{
${ }^{\text {a) }}$ Author to whom correspondence should be addressed. Electronic mail: cbaldasseroni@berkeley.edu.
}

Here, we report the temperature dependent imaging of FM domains in epitaxial FeRh thin films across the AF-FM transition on both heating and cooling by $\mathrm{x}$-ray magnetic circular dichroism (XMCD) in photoemission electron microscopy (PEEM). We show that understanding the different stages of the transition at the microscopic level enables a consistent explanation of the macroscopic magnetization behavior. The coexistence of laterally separated AF and FM phases is observed and different stages of the phase transformation are studied: nucleation, growth, and domain coalescence.

$90 \mathrm{~nm}$ thick (001) FeRh thin films were grown by magnetron sputtering from a FeRh alloy target onto (001) $\mathrm{MgO}$ substrates. The base pressure in the chamber was $8 \times 10^{-8}$ Torr. The growth was performed at a substrate temperature of $873 \mathrm{~K}$, using a DC power of $40 \mathrm{~W}$ and an argon pressure of $2 \mathrm{mTorr}$. The growth rate measured by a quartz crystal microbalance was $0.04 \mathrm{~nm} / \mathrm{s}$. One of the films was capped with $2.5 \mathrm{~nm}$ of sputtered $\mathrm{Al}$, the other was left uncapped. X-ray absorption spectroscopy showed no oxidation of $\mathrm{Fe}$ in the capped thin film and very minimal oxidation in the uncapped thin film. We have also verified by $\mathrm{x}$-ray photoelectron spectroscopy that the $\mathrm{Al}$ is not fully oxidized, leading to the conclusion that the interface of the capped film is metallic $\mathrm{Al}$ against FeRh. The thickness of the oxide formed in the uncapped film was later estimated from hard $\mathrm{x}$-ray photoemission spectroscopy to be less than $2 \mathrm{~nm}$.

The $\mathrm{CsCl}$ structure and epitaxy of the thin film were verified by $x$-ray diffraction. A $\theta-2 \theta$ geometry scan showed (001) texture out-of-plane and a $\varphi$ scan on the (101) peak 

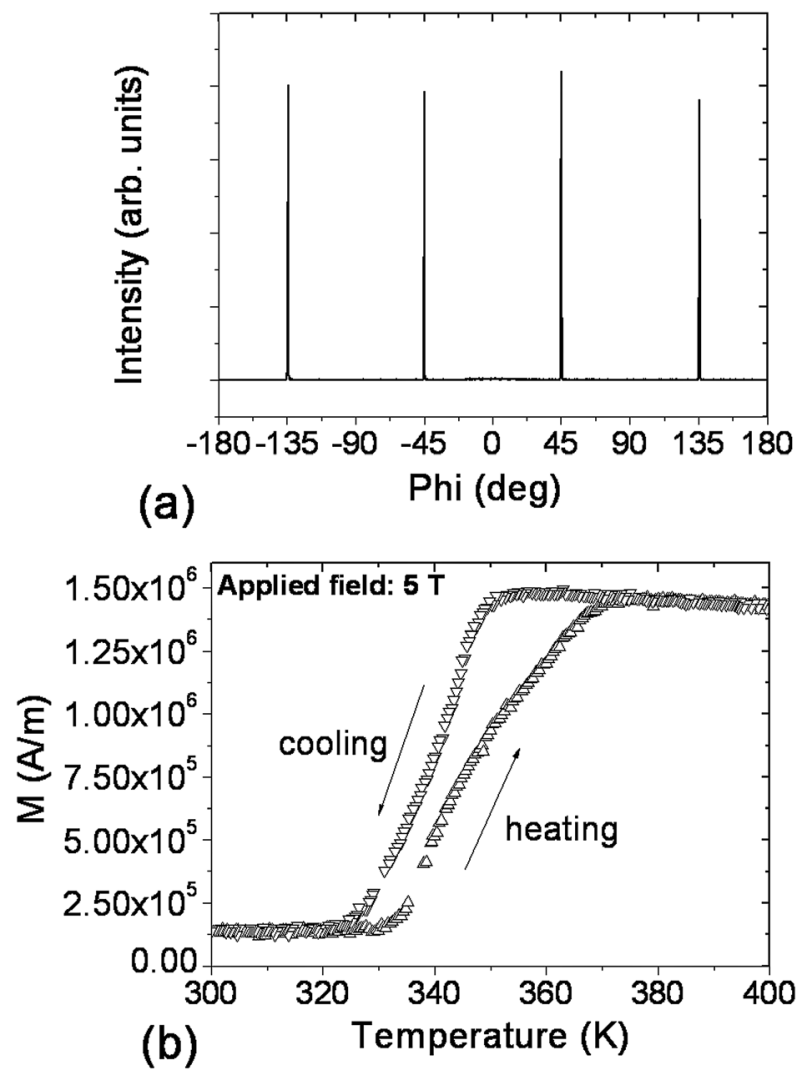

FIG. 1. Structural and magnetic characterization of uncapped FeRh thin film. (a) Phi scan of the (101) FeRh peak showing the four fold symmetry characteristic of an epitaxial growth. (b) Magnetization hysteresis loops of the uncapped FeRh thin film as a function of temperature.

showed four-fold symmetry as seen in Fig. 1(a), with the FeRh peaks rotated by $45^{\circ}$ with respect to the $\mathrm{MgO}$ (101) peak, as previously observed. ${ }^{8}$ The mosaic spread was measured to be $0.33^{\circ}$ indicating good structural order. The inplane and out-of-plane lattice parameters were measured to be $\mathrm{a}=0.2988 \mathrm{~nm}$ and $\mathrm{c}=0.2986 \mathrm{~nm}$, respectively, showing a very good agreement with the lattice parameter of bulk FeRh $(0.299 \mathrm{~nm})$ with a slight tetragonal distortion of $0.07 \%$.

The magnetic phase transition of both the capped and uncapped samples was verified by SQUID magnetometry. Figure 1(b) shows the temperature dependence of the magnetization of the uncapped sample in an applied field of $5 \mathrm{~T}$. The capped sample magnetization data were very similar, with the saturation magnetization being $10 \%$ higher. The middle of the transition occurs at $340 \mathrm{~K}$ in $5 \mathrm{~T}$, with a small hysteresis of $4 \mathrm{~K}$ between heating and cooling. The transition temperature of the first-order phase transition between $\mathrm{AF}$ and FM shifts with field according to $\mathrm{dT} / \mathrm{dH}=-8 \mathrm{~K} / \mathrm{T}$. By correcting for the temperature shift due to the field the transition is expected to occur at $380 \mathrm{~K}$ in the absence of applied magnetic field.

PEEM imaging of both samples was performed at BESSY II beamline UE49-PGM-a-SPEEM at the $\mathrm{Fe}_{3}$ edge $(705.6 \mathrm{eV})$, using low-energy secondary electrons with a probing depth of $\sim 5 \mathrm{~nm}$ (larger than the thickness of the cap and the oxide layer) ${ }^{16}$ and a spatial resolution of $\sim 30 \mathrm{~nm}$. The magnetic contrast that allows imaging of FM domains is obtained by using XMCD at the $\mathrm{Fe}_{3}$ edge. ${ }^{17}$ PEEM images were recorded with left-circularly polarized (LCP) and right- circularly polarized (RCP) x-rays. In the following images, the magnetic contrast is displayed as the difference of two PEEM images taken with opposite helicities divided by their sum, to yield the XMCD asymmetry as $A=\frac{I_{-}-I_{+}}{I_{-}+I_{+}}$. This $\mathrm{XMCD}$ asymmetry is finally proportional to the projection of the Fe magnetic moment on the incident direction of the $\mathrm{x}$-ray beam ${ }^{18}$ and is computed pixel-by-pixel. To improve the signal-to-noise ratio, each PEEM image is constructed by averaging 120 single snapshots, with each acquired over an exposure time of $3 \mathrm{~s}$. A blue-white-red colorscale was chosen in XMCD-PEEM images so as to show areas with zero asymmetry as white. The sample temperature was ramped up continuously from room temperature to $415 \mathrm{~K}$ and back, so as to record the complete transition upon heating and cooling. The temperature increased by about $5 \mathrm{~K}$ during the recording time of each PEEM image; the average temperature during the measurement is presented. Changes in magnetic contrast can generally be attributed to 3 individual factors: the magnitude of the $\mathrm{Fe}$ moments, the direction of the $\mathrm{Fe}$ moments, and the fraction of $\mathrm{Fe}$ atoms within the probing depth of ca. $5 \mathrm{~nm}$ that carry a FM moment. No external magnetic field was applied during the measurement.

Figure 2 shows representative images of both films near $\mathrm{RT}$ (AF state) and above $400 \mathrm{~K}$ (FM state) taken at the $\mathrm{Fe}_{3}$ edge. While the high temperature image in each film (Figs. 2(b) and 2(d)) shows a similar complex pattern of micron size FM domains with strong asymmetry ranging from -0.15 to 0.15 (the same order of magnitude as asymmetry of pure $\mathrm{Fe}$ at the $\mathrm{Fe}_{3}$ edge), a striking difference can be observed between the two room-temperature images shown
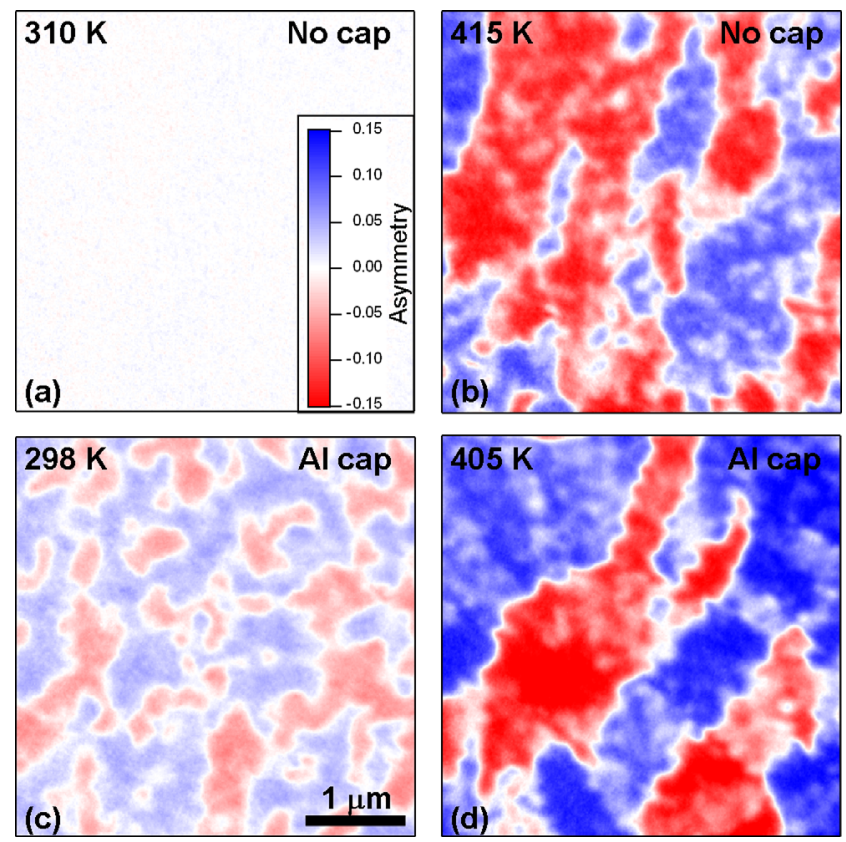

FIG. 2. Effect of capping layer and temperature on FM domains in FeRh thin film. XMCD-PEEM asymmetry images (difference between right and left polarization images divided by their sum, in zero applied magnetic field) of FeRh thin films showing FM domains in the AF and FM states (temperature of image shown in upper left corner) for the film without capping layer (No cap (a) and (b)) and the film capped with $2.5 \mathrm{~nm}$ of Al (Al cap (c) and (d)). FM domains with a positive (negative) projection of the magnetization onto the x-ray beam direction are shown in different intensities of blue (red). The asymmetry colorscale used for the 4 images is shown in (a). 
in Figs. 2(a) and 2(c). The uncapped sample shows no magnetic contrast, whereas the capped sample clearly shows FM domains, albeit with reduced contrast compared to the fully FM images. Since the oxide layer of the uncapped sample is thinner than the probing depth, the vanishing magnetic contrast of the uncapped sample is attributed to FeRh in the AF phase, while the domains of the capped sample reveal a stable FM phase at the interface with the Al capping layer. In fact, interfacial ferromagnetism at RT has been previously observed by total electron yield XMCD in FeRh thin films capped with $\mathrm{MgO}$ and Au by Ding et al ${ }^{19}$ In a subsequent study, Fan et $a l^{20}{ }^{20}$ used polarized neutron reflectometry to model the magnetic depth profile of FeRh (in a magnetic field applied in the plane of the film) capped with $\mathrm{MgO}$ at $\mathrm{RT}$ and confirmed the presence of interfacial ferromagnetism near the top interface, but with a highly reduced signal compared to that of a fully FM film at $400 \mathrm{~K}$. The stabilization of the FM phase at the interface is assumed to result from a combination of Fe deficiency and strain effects due to the cap. The thin native oxide does not have a similar effect or it is so weak as to be undetectable.

Our observation is in good agreement with these prior results. We attribute the reduced magnetic contrast in Fig. 2(c) relative to Fig. 2(d) to a possible combination of reduced atomic Fe moments and a thin FM layer compared to the probing depth of ca. $5 \mathrm{~nm}$. An alternative explanation for the reduced contrast is canting of the moments out of the plane; however, this is unlikely as it would result in some regions with increased magnetic contrast which we do not observe.

Since the presence of the Al cap generates FM domains near the interface even at RT we turn to the uncapped sample to study the transition from the fully AF to the fully FM state. The spatial resolution of the PEEM technique allows us to look at the evolution of the FM domains as a function of temperature without an external magnetic field, as shown in Fig. 3((a)-(e) heating and (f)-(j) cooling).
In an effort to relate the transition at the microscopic level to the macroscopic behavior, we calculated the integrated absolute XMCD (|XMCD|) for a circular region of interest at the center of the field of view, shown as an inset in Fig. 3(k). Figure 3(k) compares the evolution of IXMCDI as a function of the sample temperature to the temperature evolution of the macroscopic magnetization. The magnetization was recorded by SQUID magnetometry in an applied field of $5 \mathrm{~T}$ (Fig. 1) and was then corrected by an $8 \mathrm{~K} / \mathrm{T}$ shift determined experimentally so as to extrapolate the $\mathrm{M}-\mathrm{T}$ transition to zero field. The magnetization recorded by magnetometry in $5 \mathrm{~T}$ is a close approximation to the fraction of FM phase. The IXMCDI measurement includes domains and therefore is not as good a representation of the fraction of FM phase but still shows a similar hysteretic behavior to the M-T curve. In particular, the middle of the transition agrees on both curves around $380 \mathrm{~K}$. Since magnetization is the better representation of the fraction of FM phase, we relate each PEEM image to its position on the M-T hysteresis curve using letter labels in Fig. 3(k).

Figures 3(a)-3(e) thus show the nucleation and growth of FM domains and confirm the first-order character of this magnetic transition. At RT, the uncapped film has vanishing magnetic contrast (due to a fully AF surface). Upon heating, we observe the appearance of small spots of magnetic contrast, attributed to the nucleation of small FM islands in an $\mathrm{AF}$ background. The nucleation of FM islands out of the AF matrix starts near $355 \mathrm{~K}$ (Fig. 3(a)). Over a broad temperature range (about $30 \mathrm{~K}$ ), we observe the coexistence of FM and $\mathrm{AF}$ areas. $\mathrm{FM}$ islands continue to nucleate in different areas of the sample, as seen at $364 \mathrm{~K}$ and $379 \mathrm{~K}$ (Figs. 3(b) and $3(\mathrm{c})$ ). Above $380 \mathrm{~K}$, where a change of slope is observed on the magnetization curve (Fig. 3(k)), the phase transformation switches into a domain-growth regime. The number of domains stops increasing but their size starts to significantly increase. This continues over a range of $30 \mathrm{~K}$ (from $380 \mathrm{~K}$ to $410 \mathrm{~K})$. In the middle of the growth regime, at $396 \mathrm{~K}$, the
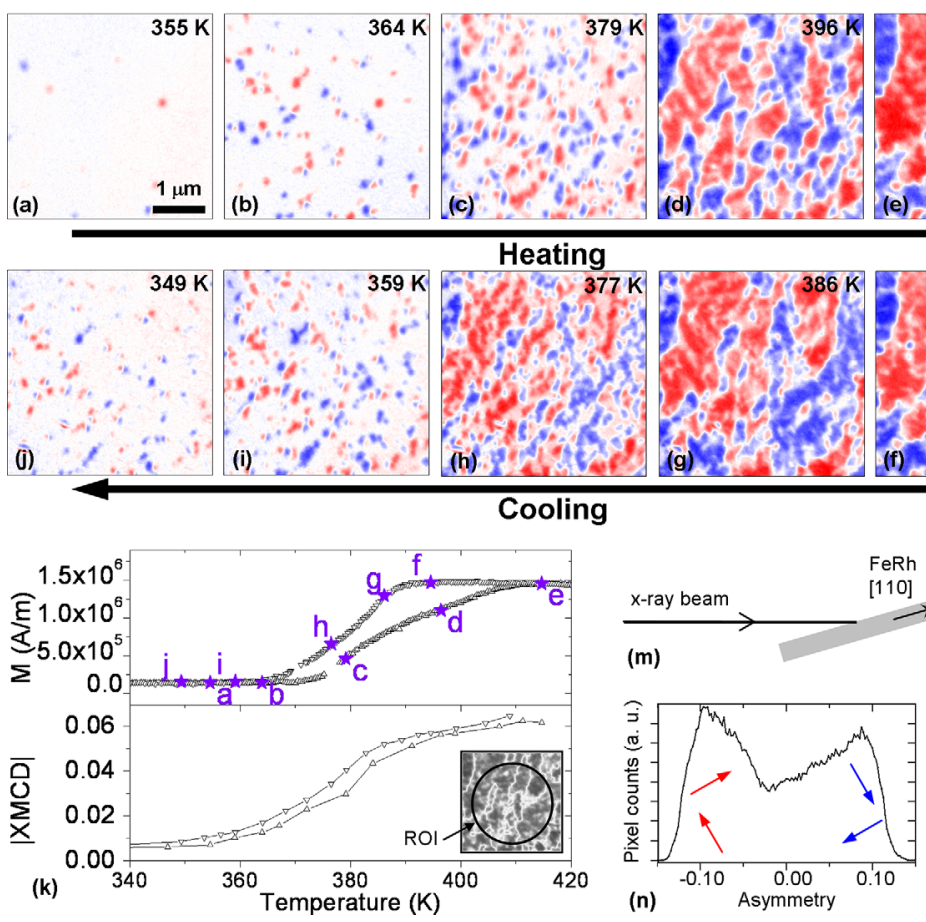

hv // FeRh [110]

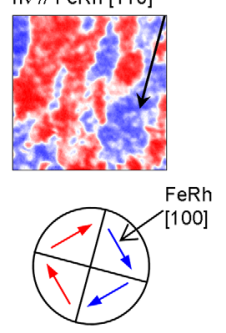

(p)
FIG. 3. Temperature evolution of FM domains in the uncapped FeRh thin film, temperature hysteresis, and FM domain configuration. (a) to (e) show heating from $\mathrm{AF}$ to FM and (f) to (j) show cooling from FM to $\mathrm{AF}$, in zero field. The asymmetry (difference between right and left polarization images divided by their sum) colorscale used for these images is from -0.15 to 0.15 . Normalized M-T hysteresis measured at $5 \mathrm{~T}$ is shown in the upper panel of $(\mathrm{k})$; the temperature axis has been corrected to zero field. Integrated absolute XMCD of a representative region of interest (ROI-shown in inset) as a function of temperature is shown in the lower panel of (k). The sample configuration with respect to the $\mathrm{x}$-ray beam and the direction of the projection of the beam in the images are shown in $(\mathrm{m})$. The distribution of asymmetry in (e) $(415 \mathrm{~K})$ is shown as a histogram in (n). The two maxima are assigned to two pairs of equivalent FeRh $\langle 100\rangle$ in-plane directions as preferred orientation of FM domains (easy axis). The four $\langle 100\rangle$ directions are sketched in (p). 
domains can be observed to have more than doubled in size (Fig. 3(d)). At $415 \mathrm{~K}$, the image shows a fully FM phase (Fig. 3(e)) with a complex pattern of domains similar to the results of magnetic force microscopy by other groups. ${ }^{14,15}$ Two stages of the phase transformation were observed, one dominated by domain nucleation at low temperature and one dominated by domain growth at high temperature, as supported by classical nucleation theory, and further confirming the first-order character of the transition.

The in-plane projection of the X-ray beam is along the [110] direction of the FeRh film, as shown in Fig. 3(m), where the beam direction is indicated in the PEEM image taken at $415 \mathrm{~K}$. As noted above, XMCD-PEEM is sensitive to the component of magnetization parallel to the beam. Magnetization directions with the same projection have the same contrast. The histogram of asymmetry distribution shown in Fig. 3(n) exhibits two maxima. Because of the inplane cubic symmetry of the FeRh film, magnetocrystalline anisotropy should result in four equivalent preferred directions, at $90^{\circ}$ angles from each other. The $\langle 100\rangle$ directions are at $45^{\circ}$ from the beam direction and therefore their projections are equivalent two by two, as shown on Fig. 3(p). This is compatible with the two maxima of the histogram. If domains were $\langle 110\rangle$ type, there would be a maximum at zero asymmetry, and if the film had no anisotropy there would be no peak in the histogram. Therefore, the FM domain structure consists of four types of in-plane domains along $\langle 100\rangle$ easy axes separated by $90^{\circ}$ domain walls.

Upon cooling, the reverse transition from FM to AF is observed. At $395 \mathrm{~K}$, the sample is still fully FM, in agreement with the M-T hysteresis. From $395 \mathrm{~K}$ to $377 \mathrm{~K}$, the FM domains decrease in size (Figs. 3(f) to 3(h)). From $377 \mathrm{~K}$ to $359 \mathrm{~K}$, the fraction of $\mathrm{AF}$ phase (white regions) increases, indicating the nucleation and growth of the AF phase. At 359 K (Fig. 3(i)), the coexistence of the two magnetic phases is again clearly seen and the hysteretic behavior of the transition is evidenced by the larger number of FM nuclei compared to the PEEM image taken at $364 \mathrm{~K}$ upon heating (Fig. 3(b)). Finally, a small fraction of the thin film is still FM at $349 \mathrm{~K}$ as seen on Fig. 3(j) but is gone completely by $310 \mathrm{~K}$ (Fig. 2(a)). Comparison of the beginning of the FM to AF transition at $364 \mathrm{~K}$ upon heating (Fig. 3(b)) to the end of the FM to AF transition at $349 \mathrm{~K}$ upon cooling (Fig. 3(j)) shows that the majority of the nucleation sites identified upon heating are conserved upon cooling, evidence of heterogeneous nucleation as inferred from macroscopic magnetization measurements by Maat et al. ${ }^{8}$ The transition at the surface of the film seen in PEEM thus follows very closely the temperature hysteresis of the entire film as measured by magnetometry upon heating and cooling.

We now turn to the details of the nucleation phase. In the early stage of the nucleation phase, we observe single domain FM nuclei with a diameter as small as $100 \mathrm{~nm}$. Four different representative single domain nuclei are indicated in the inset in Fig. 4(a). The nuclei were chosen to show the extent of the nucleation phase over a wide temperature range. In the XMCD-PEEM image taken at $372 \mathrm{~K}$, all four nuclei are present but they actually nucleated at different temperatures as shown by plotting the absolute asymmetry of the center of each nucleus $(6 \times 6$ pixels, $60 \mathrm{~nm}$ wide $)$ as a
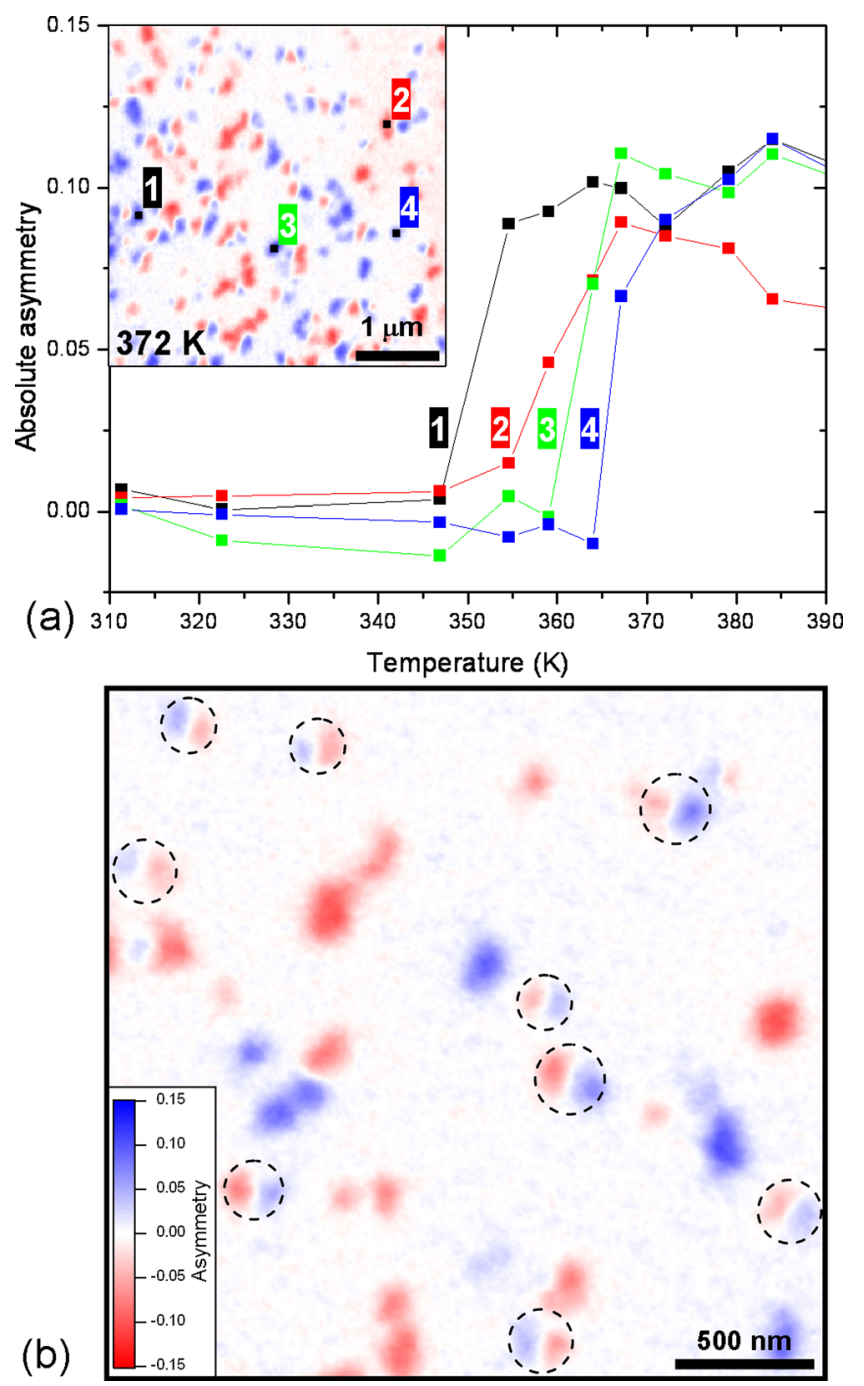

FIG. 4. (a) Temperature evolution of absolute asymmetry of 4 different local areas ( 6 pixels $\times 6$ pixels) showing the sharp local increase in magnetic contrast at the single domain nucleus scale and the spread of the nucleation regime over about $25 \mathrm{~K}$. The location of the nuclei is indicated in the inset (XMCD-PEEM asymmetry image at $372 \mathrm{~K}$ ). While islands initially nucleate as single domains, they then break into several domains upon increasing size. (b) An enlargement of the XMCD-PEEM image from Fig. 3(b) taken at $364 \mathrm{~K}$ showing FM nuclei in AF matrix in an uncapped FeRh thin film. Several nuclei with diameter of $200 \mathrm{~nm}$ or above are indicated by dotted circles and show flux closure patterns as seen by red and blue lobes.

function of temperature in Fig. 4(a). The temperature spread is attributed to different heterogeneous nucleation sites. The activation energy $\Delta G_{b}$ for heterogeneous nucleation at a defect (e.g., grain boundary) can be written as $\Delta G_{b}=f \Delta G_{H}$, with $f$ a shape factor dependent on the nature of the defect, and $\Delta G_{H}$ the activation energy for homogeneous nucleation. Different types of defects, for example, low-angle grain boundaries with varying angles, result in a range of shape factors, therefore a range of activation energies and ultimately a range of nucleation temperatures. The four different nucleation sites indicated in Fig. 4 show that the nucleation temperature range extends over $25 \mathrm{~K}$, in good agreement with the observation of the nucleation phase from $355 \mathrm{~K}$ to $380 \mathrm{~K}$ in Fig. 3. When the probing area is the size of a single nucleus, the transition between the two distinct phases is observed to be quite abrupt. The width of the local transition is less than $10 \mathrm{~K}$, as indicated by a rapid increase of 

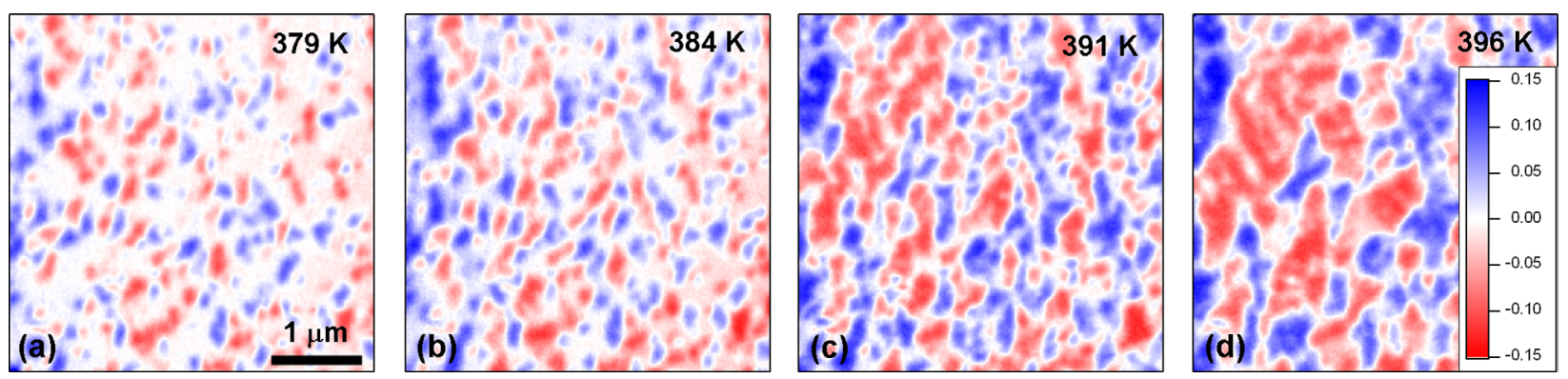

FIG. 5. XMCD-PEEM asymmetry images showing the transition from nucleation regime to growth regime in FeRh thin film between $379 \mathrm{~K}$ (same as Fig. 3(c)) and $396 \mathrm{~K}$ (same as Fig. 3(d)). The coalescence of FM domains is seen between (b) $384 \mathrm{~K}$ and (c) $391 \mathrm{~K}$.

asymmetry from 0 to 0.10 within $10 \mathrm{~K}$ or less. Note, however, that the temperature was ramped continuously during the recording time of each PEEM image; this temperature ramp results in a $5 \mathrm{~K}$ temperature increase during the recording of each data point on Fig. 4(a), which becomes convoluted into the local transition temperature spread. The local transition may therefore be as sharp as $5 \mathrm{~K}$. By contrast, the overall width of the transition seen in Fig. 3(k) results from an average over the entire sample, thereby broadening the transition to the $25 \mathrm{~K}$ of the nucleation phase, as well as additional broadening of about $30 \mathrm{~K}$ from the growth phase.

Figure 4(b) is an enlargement of an area of the PEEM image taken at $364 \mathrm{~K}$ (shown in Fig. 3(b)). While initially the FM nuclei form single domains many of the freshly nucleated FM islands later minimize their magnetostatic energy by formation of flux closure patterns, seen as red and blue lobes in Fig. 4(b). These flux closure patterns can be four-domain patterns $(\langle 100\rangle$ type, as shown in Fig. 3(p)) or vortex patterns with a continuously flowing magnetization and no domain walls. Both would appear the same in these images.

The drastic change in domains between $379 \mathrm{~K}$ and $396 \mathrm{~K}$ upon heating (Figs. 3(c) and 3(d)) shows the transition from FM domain nucleation to domain growth. Figure 5 shows this transition in more detail; the magnetic domain morphology changes from numerous small domains to a smaller number of larger domains. By looking at intermediate steps, we see the phenomenon of coalescence. While at $379 \mathrm{~K}$ many FM islands form flux closure patterns, between $384 \mathrm{~K}$ (Fig. 5(b)) and $391 \mathrm{~K}$ (Fig. 5(c)) the percolation limit is reached so that the exchange interaction energy favors the formation of larger domains, i.e., small islands start to overlap and coalesce into larger FM areas. The small second increase in the slope of the M-T curve (Fig. $3(\mathrm{k})$ ) around $388 \mathrm{~K}$ is attributed to the start of the coalescence between $384 \mathrm{~K}$ and $391 \mathrm{~K}$.

In summary, FM domains were imaged near the surface of high-quality FeRh thin films. The sample capped with $\mathrm{Al}$ showed interfacial FM domains which are stable at room temperature, while the bulk of the film is AF. An identical film with no cap has no observable surface FM domains at room temperature and is shown to follow the expected transition as measured by magnetometry. Nucleation, growth, and coalescence stages were identified in the phase transformation mechanism from AF to FM. The coexistence of FM and AF phases inherent to a first-order phase transition was observed in the early stages of the transition and small FM nuclei were seen to form flux closure patterns. This study of the transition at the microscopic level has thus led to a better understanding of the true dynamics and the breadth of the macroscopically observed transition.

This work was supported by the Director, Office of Science, Office of Basic Energy Sciences, Materials Sciences and Engineering Division, of the U.S. Department of Energy (DOE) under Contract No. DE-AC02-05CH11231. Research at Stanford was supported through the Stanford Institute for Materials and Energy Science (SIMES) and the LCLS by the US Department of Energy, Office of Basic Energy Sciences.

${ }^{1}$ M. Fallot and R. Hocart, Rev. Sci. 77, 498 (1939).

${ }^{2}$ F. de Bergevin and L. Muldawer, Compt. Rend. 252, 1347 (1961).

${ }^{3}$ J. S. Kouvel and C. C. Hartelius, J. Appl. Phys. 33, 1343 (1962).

${ }^{4}$ L. J. Swartzendruber, Bull. Alloy Phase Diagrams 5, 456 (1984).

${ }^{5}$ L. M. Sandratskii and P. Mavropoulos, Phys. Rev. B 83, 174408 (2011).

${ }^{6}$ M. Manekar and S. B. Roy, J. Phys. D: Appl. Phys. 41, 192004 (2008).

${ }^{7}$ M. R. Ibarra and P. A. Algarabel, Phys. Rev. B 50, 4196 (1994).

${ }^{8}$ S. Maat, J.-U. Thiele, and E. E. Fullerton, Phys. Rev. B 72, 214432 (2005).

${ }^{9}$ J. Cao, N. T. Nam, S. Inoue, Y. Y. K. Hnin, N. N. Phuoc, and T. Suzuki, J. Appl. Phys. 103, 07F501 (2008).

${ }^{10}$ M. Sharma, H. M. Aarbogh, J.-U. Thiele, S. Maat, E. E. Fullerton, and C. Leighton, J. Appl. Phys. 109, 083913 (2011).

${ }^{11}$ N. T. Nam, W. Lu, and T. Suzuki, J. Appl. Phys. 105, 07D708 (2009).

${ }^{12}$ J.-U. Thiele, S. Maat, and E. E. Fullerton, Appl. Phys. Lett. 82, 2859 (2003).

${ }^{13}$ E. F. Kneller and R. Hawig, IEEE Trans. Magn. 27, 3588 (1991).

${ }^{14}$ M. Manekar, C. Mukherjee, and S. B. Roy, Europhys. Lett. 80, 17004 (2007).

${ }^{15}$ Y. Yokoyama, M. Usukura, S. Yuasa, Y. Suzuki, H. Miyajima, and T. Katayama, J. Magn. Magn. Mater. 177-181, 181 (1998).

${ }^{16}$ B. H. Frazer, B. Gilbert, B. R. Sonderegger, and G. De Stasio, Surf. Sci. 537, 161 (2003).

${ }^{17}$ G. Schütz, W. Wagner, W. Wilhelm, P. Kienle, R. Zeller, R. Frahm, and G. Materlik, Phys. Rev. Lett. 58, 737 (1987).

${ }^{18}$ C. M. Schneider and G. Schönhense, Rep. Prog. Phys. 65, 1785 (2002).

${ }^{19}$ Y. Ding, D. A. Arena, J. Dovrak, M. Ali, C. J. Kinane, C. H. Marrows, B. J. Hickey, and L. H. Lewis, J. Appl. Phys. 103, 07B515 (2008).

${ }^{20}$ R. Fan, C. J. Kinane, T. R. Charlton, R. Dorner, M. Ali, M. A. de Vries, R. M. D. Brydson, C. H. Marrows, B. J. Hickey, D. A. Arena, B. K. Tanner, G. Nisbet, and S. Langridge, Phys. Rev. B 82, 184418 (2010). 